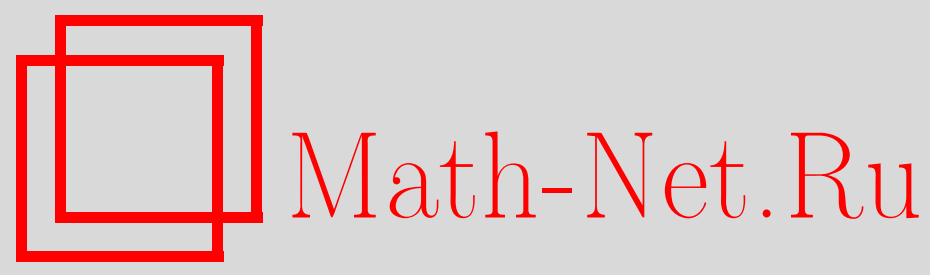

С. А. Кудрявцев, О чисто логтерминальных раздутиях, $M a$ тем. заметки, 2001, том 69, выпуск 6, 892-898

DOI: https://doi.org/10.4213/mzm702

Использование Общероссийского математического портала Math-Net.Ru подразумевает, что вы прочитали и согласны с пользовательским соглашением http://www.mathnet.ru/rus/agreement

Параметры загрузки:

IP : 54.80 .73 .141

26 апреля 2023 г., 03:16:47

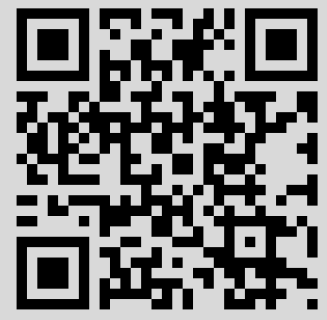




\section{О ЧИСТО ЛОГТЕРМИНАЛЬНЫХ РАЗДУТИЯХ}

\section{С. А. Кудрявцев}

Доказывается существование чисто логтерминального раздутия для логтерминальной особенности и критерий слабой исключительности особенности.

Библиограбфия: 9 названий.

Введение. Основная цель этой заметки - доказать некоторые результаты работы [1] для не $\mathbb{Q}$-факториального случая, а именно теорему о существовании чисто логтерминального раздутия (теорема 1.5) и критерий слабой исключительности особенности (теорема 2.1). Эти раздутия позволяют нам применить индуктивньй метод Шокурова [2] для изучения особенностей или, более общо, для стягиваний алгебраических многообразий. Он позволяет свести вопросы о структуре, дополняемости и исключительности особенности к единственному исключительному дивизору раздутия. Для $\mathbb{Q}$-факториальных особенностей чисто логтерминальное раздутие - это единственное раздутие, позволяющее в общем случае продолжить дополнение с исключительного дивизора на все многообразие (замечание 1.3), а для не $\mathbb{Q}-$ факториальных логтерминальных особенностей такие раздутия будут отличаться от чисто логтерминального раздутия на малое флоповое стягивание (следствие 1.13). При изучении произвольных $\mathbb{Q}$-горенштейновых особенностей практически невозможно отделить класс $\mathbb{Q}$ факториальных особенностей от остальных. Поэтому приходится пользоваться теоремами и конструкциями, которые верны в общем случае. Данная работа уточняет результаты статьи [1] о индуктивном методе изучения любых логканонических особенностей.

Автор очень признателен В. А. Исковских и Ю. Г. Прохорову за полезные обсуждения и постоянный интерес к работе.

1. Чисто логтерминальные раздутия и их свойства. В дальнейшем все многообразия являются алгебраическими и рассматриваются над полем комплексных чисел $\mathbb{C}$. Полученные результаты также верны и в случае комплексных пространств. Основные определения, обозначения и понятия приведены в [1], [3], [4]. Строго логканоническими особенностями называются логканонические, но не логтерминальные особенности.

Работа выполнена при частичной поддержке Российского фонда фундаментальных исследований, грант № 99-01-01132, программы “Ведущие научные шшколы”, грант № 96-15-96146, фонда INTAS, гранты INTAS-OPEN № 93-2805 и № 97-2072. 
ОПРЕдЕлЕниЕ 1.1. Пусть $X$ - нормальное логканоническое алгебраическое многообразие, и пусть $f: Y \rightarrow X$ - некоторое его раздутие. Предположим, что исключительное множество $f$ состоит только из одного неприводимого дивизора $E(\operatorname{Exc}(f)=E)$. Тогда $f:(Y, E) \rightarrow X$ назьвается чисто логтерминальным раздутием, если дивизор $K_{Y}+E$ чисто логтерминален и $-E f$-обилен.

ЗАмЕчАниЕ 1.2. В определении 1.1 неявно требуется, чтобы дивизор $E$ был $\mathbb{Q}$ Картье. Отсюда следует, что многообразие $Y$ имеет $\mathbb{Q}$-горенштейновые особенности.

ЗАМЕчАНИЕ 1.3. В обозначениях определения 1.1 имеем следующие свойства чисто логтерминальных раздутий.

1. Если многообразие $X$ имеет логтерминальные особенности, то дивизор $-\left(K_{Y}+E\right)$ $f$-обилен. Действительно, это следует из численной эквивалентности $K_{Y}+E \equiv$ $(a(E, 0)+1) E$ над $X$.

2. Если многообразие $X$ имеет строго логканонические особенности, то $a(E, 0)=-1$.

$3[1$, п. 2.2]. Если многообразие $X$ является $\mathbb{Q}$-ракториальным, то $Y$ тоже $\mathbb{Q}$-факториально и $\rho(Y / X)=1$. Отсюда следует, что в определении 1.1 для $\mathbb{Q}$ факториального случая, можно не требовать относительную обильность дивизора $-E$, так как она всегда имеет место. Напомним также, что каждая компонента исключительного множества любого бирационального стягивания в $\mathbb{Q}$-факториальную точку имеет коразмерность 1 .

4. По обращению присоединения дивизор $K_{E}+\operatorname{Diff}_{E}(0)$ логтерминален по Кавамате. Любое его $n$-дополнение продолжается до $n$-дополнения $K_{Y}+E$, следовательно, $K_{X}$ тоже $n$-дополняем $[1$, п. 2.8].

$5\left[1\right.$, п. 2.2]. Пусть $f_{i}:\left(Y_{i}, E_{i}\right) \rightarrow(X \ni P)$ - два чисто логтерминальных раздутия. Если $E_{1}$ и $E_{2}$ определяют одно и тоже дискретное нормирование поля функций $\mathscr{K}(X)$, то раздутия $f_{1}$ и $f_{2}$ изоморфны.

ПроьлемА 1.4. Описать класс слабых поверхностей лог-Дель Пецо, $\mathbb{P}^{1}$ (и эллиптических) расслоений в общей точке, которые могут быть реализованы как исключительные дивизоры чисто логтерминальньх раздутий терминальных, канонических, $\varepsilon$-логтерминальных, строго логканонических особенностей.

Существование чисто логтерминального раздутия для логтерминальной особенности следует из следующей теоремы.

ТЕОремА 1.5. Пусть $X$-многообразие с логтерминальными особенностями, $и$ пусть $D \neq 0$ - граница на $X$ такая, что пара $(X, D)$ является логканоничной, но не чисто логтерминальной. Предположим, что верна лог-программа минимальных моделей. (При $\operatorname{dim} X \leqslant 3$ это так.) тогда существует раздутие $f: Y \rightarrow X$ со следующими свойствами:

1) исключительное мнохсество морфизма $f$ состоит только из одного неприводимого дивизора $E(\operatorname{Exc}(f)=E)$;

2) дивизор $K_{Y}+E+D_{Y}=f^{*}\left(K_{X}+D\right)$ логканоничен;

3) дивизор $K_{Y}+E+(1-\varepsilon) D_{Y}$ чисто логтерминален и антиобилен над $X$ для любых $\varepsilon>0$

4) если многообразие $X \mathbb{Q}$-факториально, то $Y$ тоже $\mathbb{Q}$-факториально и $\rho(Y / X)=1$. 
ДокАЗАТЕЛЬСтво. Для удобства читателей напомним доказательство этой теоремы для $\mathbb{Q}$-факториальных особенностей $[1$, п. 2.9]. Пусть $g: \widehat{Z} \rightarrow X$ - минимальное логтерминальное разрешение с приведенным исключительным дивизором $\widehat{E}=\sum \widehat{E}_{i}[3$, п. 17.10], [5, п. 9.1]. По определению такого разрешения многообразие $\widehat{Z} \mathbb{Q}$-факториально и дивизор $K_{\widehat{Z}}+\widehat{E}+D_{\widehat{Z}}=g^{*}\left(K_{X}+D\right)$ является дивизориально логтерминальным. Так как $X$ имеет логтерминальные особенности, то дивизор

$$
K_{\widehat{Z}}+\widehat{E}=g^{*} K_{X}+\sum(a(E, 0)+1) \widehat{E}_{i}
$$

не может быть $g$-численно эффективным по численньм свойствам сдутий [5, п. 1.1]. Применим $\left(K_{\widehat{Z}}+\widehat{E}\right)$-программу минимальных моделей. На последнем шаге имеем стягивание $g^{\prime}: \widetilde{Z} \rightarrow X$ (см. диаграмму (1)). Дивизор $K_{\widetilde{Z}}+\widetilde{E}+D_{\widetilde{Z}}=g^{*}\left(K_{X}+D\right)$, где $\widetilde{E}-$ неприводимьй дивизор, логканоничен. Так как $K_{\widetilde{Z}}+\widetilde{E}$ чисто логтерминальньй дивизор, то пара $\left(\widetilde{Z}, \widetilde{E}+(1-\varepsilon) D_{\widetilde{Z}}\right)$ чисто логтерминальна для любых $\varepsilon>0$.

Если многообразие $X \quad \mathbb{Q}$-факториально, то согласно замечанию $1.3 \operatorname{Exc}\left(g^{\prime}\right)=\widetilde{E}$, $\rho(\widetilde{Z} / X)=1$ и $-\widetilde{E} g^{\prime}$-обилен, поэтому $g^{\prime}$ - искомое раздутие:

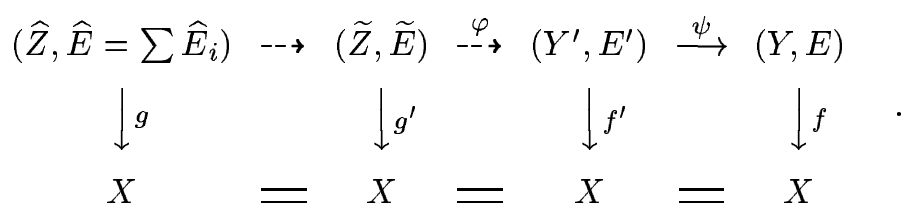

Предположим, что $\operatorname{Exc}\left(g^{\prime}\right)=\widetilde{E} \cup \Delta$, где $\Delta \neq \varnothing$ и $\operatorname{codim}_{\widetilde{Z}} \Delta \geqslant 2$. Из численной эквивалентности $K_{\widetilde{Z}}+D_{\widetilde{Z}} \equiv-\widetilde{E}$ над $X$ следует, что дивизор $K_{\widetilde{Z}}+D_{\widetilde{Z}}$ не может быть $g^{\prime}$-численно эффективньг и он неотрицателен на кривых, лежащих на $\widetilde{E}$. Применим $\left(K_{\widetilde{Z}}+D_{\widetilde{Z}}\right)$-программу минимальных моделей. В итоге получим стягивание $f^{\prime}: Y^{\prime} \rightarrow X$, у которого $\operatorname{Exc}\left(f^{\prime}\right)=E^{\prime}$, где $E^{\prime}$ - неприводимьй дивизор. Заметим, что бирациональное отображение $\varphi$ раскладьвается в произведение лог-флипов. Дивизор $K_{Y^{\prime}}+E^{\prime}+$ $(1-\varepsilon) D_{Y^{\prime}}$ чисто логтерминален для любых $\varepsilon>0$. Если $-E^{\prime} f^{\prime}$-обилен, то $f^{\prime}-$ искомое раздутие.

Пусть дивизор $-E^{\prime}$ не $f^{\prime}$-обилен. Так как $-E^{\prime} f^{\prime}$-численно эффективен и

$$
K_{Y^{\prime}}+E^{\prime}=f^{\prime *} K_{X}+(a(E, 0)+1) E^{\prime},
$$

где $a(E, 0)+1>0$, то $-\left(K_{Y^{\prime}}+E^{\prime}\right) f^{\prime}$-численно эффективен. По теореме о свободе [6, п. 3.1.2] для логтерминального по Кавамате дивизора $K_{Y^{\prime}}+(1-\delta) E^{\prime}$, где $0<\delta \ll 1$, для больших $n$ линейная система $\left|-n\left(K_{Y^{\prime}}+E^{\prime}\right)\right|$ свободна над $X$. Она задает мальй бирациональньй морфизм $\psi:\left(Y^{\prime}, E^{\prime}\right) \rightarrow(Y, E)$. Пусть $C$ - стягиваемая кривая. Так как $\left(K_{Y^{\prime}}+E^{\prime}\right) \cdot C=0$, то $E^{\prime} \cdot C=0$ и $K_{Y^{\prime}} \cdot C=0$. Итак, морфизм $\psi$ стягивает в точности те кривые, которые пересекаются с дивизором $E^{\prime}$ по нулю. Полученное раздутие $f:(Y, E) \rightarrow X$, очевидно, является искомым. 
ОПРЕДЕЛЕнИЕ 1.6. Логканоническая особенность $(X \ni P)$ назьвается слабо исключительной, если для нее существует ровно одно чисто логтерминальное раздутие (с точностью до изоморфизма). Логканоническая пара $(X, D)$, где дивизор $D$ - гранища, назьвается исключительной, если существует не более одного исключительного дивизора $E$ с дискрепантностью $a(E, D)=-1$. Логканоническая особенность $(X \ni P)$ назьвается исключительной, если все логканонические пары $(X, D)$ являются исключительньми.

Для следующего следствия из теоремы 1.5 также нужна лог-программа минимальных моделей.

СлЕДСТВИЕ 1.7. Пусть $f:(Y, E) \rightarrow(X \ni P)$ - чисто логтерминальное раздутие логтерминальной особенности. Предположим, что $\operatorname{dim} f(E) \geqslant 1 ;$ тогда существует другое чисто логтерминальное раздутие $(X \ni P)$, поэтому особенность не слабо исключительна.

ДокАЗАТЕЛЬСТво. Возьмем два гиперплоских сечения $H_{1}$ и $H_{2}$, проходящих через точку $P$ и не содержащих $f(E)$. Пусть $c>0$-логканонический порог дивизора $H_{1}+H_{2}$. Тогда дивизор $K_{X}+c\left(H_{1}+H_{2}\right)$ не чисто логтерминален. Множество $f(E)$ отлично от $L C S\left(X, c\left(H_{1}+H_{2}\right)\right)$. Применяя теорему 1.5 к $\left(X, c\left(H_{1}+H_{2}\right)\right)$, получаем требуемое.

ПРЕДЛОЖЕНИЕ 1.8. Пусть $f:(Y, E) \rightarrow(X \ni P)$ - чисто логтерминальное раздутие строго логканонической особенности. Предположим, что $\operatorname{dim} f(E) \geqslant 1$. Тогда $(X \ni P)$ - не исключительная особенность.

ДокАЗАТЕЛЬСТвО. Как и в доказательстве следствия 1.7 , существует дивизор $D$ такой, что пара $(X, D)$ логканонична, но не чисто логтерминальна и множество $f(E)$ отлично от минимального центра $L C S(X, D)$. Противоречие с определением исключительности.

Для доказательства необходимости в следующей теореме нужна лог-программа минимальных моделей.

Теорема 1.9. Пусть $(X \ni P)$ - строго логканоническая особенность. Тогда

1) если существует чисто логтерминальное раздутие, то оно единственно (с точностью до изоморфизма);

2) особенность является исключительной тогда и только тогда, когда существует чисто логтерминальное раздутие $f:(Y, E) \rightarrow(X \ni P)$ такое, что $f(E)=P$.

ДокАЗАТЕЛЬСтво. Первое утверждение немедленно следует из свойств 2 и 5 из замечания 1.3. Докажем вторую часть теоремы.

Необходимость. Построение чисто логтерминального раздутия повторяет доказательство теоремы 1.5. Пусть $g^{\prime}: \widetilde{Z} \rightarrow X$ - минимальное логтерминальное разрешение с приведенным исключительным дивизором $\widetilde{E}=\sum \widetilde{E}_{i}$. По определению такого разрешения многообразие $\widetilde{Z} \mathbb{Q}$ фракториально и дивизор $K_{\widetilde{Z}}+\widetilde{E}=g^{\prime *} K_{X}$ дивизориально логтерминален. Так как особенность исключительна, дивизор $\widetilde{E}$ неприводим и поэтому $K_{\widetilde{Z}}+\widetilde{E}$ чисто логтерминален. $\operatorname{Ecли~} \operatorname{Exc}\left(g^{\prime}\right)=\widetilde{E} \cup \Delta$, где $\Delta \neq \varnothing$ и $\operatorname{codim}_{\widetilde{Z}} \Delta \geqslant 2$, то применим $K_{\widetilde{Z}}$-программу минимальных моделей. В итоге получим стягивание $f^{\prime}: Y^{\prime} \rightarrow X$, 
у которого $\operatorname{Exc}\left(f^{\prime}\right)=E^{\prime}$, где $E^{\prime}$ - неприводимьй дивизор. Дивизор $K_{Y^{\prime}}+E^{\prime}$ останется чисто логтерминальным. Если $-E^{\prime} f^{\prime}$-обилен, то $f^{\prime}$ - искомое раздутие. Пусть $-E^{\prime}$ не $f^{\prime}$-обилен. Так как $-E^{\prime} f^{\prime}$-численно эффективен и $K_{Y^{\prime}} \equiv-E^{\prime}$ над $X$, то $K_{Y^{\prime}} f^{\prime}$-численно эффективен. По теореме о свободе [6, п. 3.1.2] для больших $n$ линейная система $\left|n K_{Y^{\prime}}\right|$ свободна над $X$. Она задает малый бирациональньй морфизм $h:\left(Y^{\prime}, E^{\prime}\right) \rightarrow(Y, E)$. Полученное раздутие $f:(Y, E) \rightarrow X$ является чисто логтерминальным, так как $K_{Y} f$-обилен. Из предложения $1.8 f(E)=P$.

Достаточность. Заметим, что $E$ - единственньй исключительный дивизор с дискрепантностью $a(E, 0)=-1$. Пусть $(X, D)$ - произвольная логканоническая пара, так как $f(E)=P$, то $D=0$.

СлЕДСТВИЕ 1.10. Для строго логканонической исключительной особенности минимальный индекс дополняемости совпадает с индексом горенштейновости особенности.

ЗАмЕчАниЕ 1.11. Для трехмерных логканонических особенностей минимальньй индекс дополняемости ограничен [2, п. 7.1]. Гипотетически, он не более 66. Для трехмерных исключительных строго логканонических особенностей это доказано в работах [7] и [8]. Для неисключительных неизолированных особенностей индекс горенштейновости неограничен [8, п. 5.1].

СлЕДСТВИЕ $1.12[9$, п. 2.4]. Исключительная особенность является слабо исключительной.

ДоКАЗАТЕЛЬСтво. Существование чисто логтерминального раздутия следует из теорем 1.5 и 1.9 , а по [9, п. 2.4] оно единственно.

Из доказательств теорем 1.5 и 1.9 имеем следующее

СлЕДСТВИЕ 1.13. Если в определении 1.1 не требовать относительную обильность дивизора -E, то такие раздутия могут отличаться от чисто логтерминального раздутия только на малое флоповое стягивание.

2. Критерий слабой исключительности особенности. Для доказательства импликации $(3) \Longrightarrow(1)$ в следующей теореме нужна лог-программа минимальньх моделей.

ТЕОрема 2.1. Пусть $(X \ni P)$ - логтерминальная особенность, и пусть $f:(Y, E)$ $\rightarrow X-$ ее чисто логтерминальное раздутие. Тогда следующие условия әквивалентны:

1) особенность $(X \ni P)$ не слабо исключительная;

2) существует эффективный $\mathbb{Q}$-дивизор $D \geqslant \operatorname{Diff}_{E}(0)$ такой, что $-\left(K_{E}+D\right)$ - обильный дивизор и пара $(E, D)$ не логтерминальна по Кавамате;

3) существует әффективный $\mathbb{Q}$-дивизор $D \geqslant \operatorname{Diff}_{E}(0)$ такой, что $-\left(K_{E}+D\right)$ - обильный дивизор и пара $(E, D)$ не логканонична.

ДоказАтЕльство. Доказательства импликаций $(1) \Longrightarrow(2)$ и $(2) \Longrightarrow(3)$ см. в $[1$, п. 4.3]. Осталось доказать $(3) \Longrightarrow(1)$. По следствию 1.7 можно считать, что $f(E)=P$. 
Из доказательства $[1$, теорема 4.3] следует сушествование эффективного $\mathbb{Q}-$ Картье дивизора $B=\sum b_{i} B_{i}$ такого, что дивизор $K_{Y}+E+B$ логканоничен, но не чисто логтерминален и антиобилен над $X$. Пусть $H$ - очень обильньй дивизор, содержащий минимальный центр $L C S(Y, E+B)$. Пусть $\varepsilon>0$ - достаточно малое рациональное число такое, что дивизор $-\left(K_{Y}+E+B+\varepsilon H\right) f$-обилен. Заменяя $B$ на дивизор $c(B+\varepsilon H)$, можем добиться, что все $b_{i}<1$ ( $c<1$, так как $H$ содержит минимальньй центр) и все другие условия сохранятся. Положим $L=f(B)$. Так как дивизор $-\left(K_{Y}+E+B\right) f$-обилен и все $b_{i}<1$, то логканонический порог $c^{\prime}$ пары $(X, L)$ строго больше единицы. Если пара $\left(X, c^{\prime} L\right)$ чисто логтерминальна, то добавим эффективньй $\mathbb{Q}-$ Картье дивизор $L^{\prime}$ и будем считать, что пара $\left(X, c^{\prime} L+L^{\prime}\right)$ логканонична, но не чисто логтерминальна. Применяя к ней теорему 1.5 , получаем чисто логтерминальное раздутие $f^{\prime}:\left(Y^{\prime}, E^{\prime}\right) \rightarrow X$. Раздутия $f$ и $f^{\prime}$ не изоморфны, так как дивизор $K_{Y}+E+c^{\prime} L_{Y}+L_{Y}^{\prime}$ не логканоничен $\left(c^{\prime}>1\right)$.

ПримеР 2.4 [1, п. 4.7], [4, п. 6.4]. Двухмерные слабо исключительные логтерминальные особенности - это в точности особенности типов $\mathbb{D}_{n}, \mathbb{E}_{6}, \mathbb{E}_{7}, \mathbb{E}_{8}$. Среди них только особенности типа $\mathbb{D}_{n}$ не являются исключительньми. Двухмерные слабо исключительные строго логканонические особенности (по теореме 1.9 они исключительны) - это в точности особенности следующих типов: простые эллиптические особенности, особенности типа $\widetilde{\mathbb{D}}_{4}$ с графом минимального разрешения на рис. 1 и особенности типов $\widetilde{\mathbb{E}}_{6}, \widetilde{\mathbb{E}}_{7}, \widetilde{\mathbb{E}}_{8}$ с графом минимального разрешения на рис. 2 , где $\left(n_{1}, n_{2}, n_{3}\right)=(3,3,3)$, $(2,4,4)$ или $(2,3,6)$ соответственно.

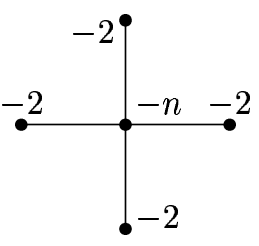

Рис. 1

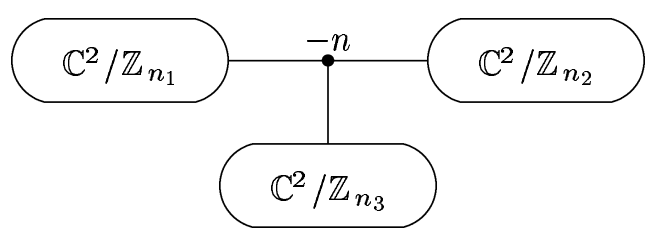

РИС. 2

ЗАмечАниЕ 2.3. Трехмерная терминальная особенность не слабо исключительна [1, п. 4.8].

ПРИмеР 2.2. При $n \geqslant 3$ рассмотрим $n$-мерную каноническую гиперповерхностную особенность

$$
\left(x_{1}^{n}+\cdots+x_{n}^{n}+x_{n+1}^{n+1}=0\right) \subset\left(\mathbb{C}^{n+1}, 0\right) .
$$

Взвешенное раздутие $\mathbb{C}^{n+1}$ с весами $(n+1, \ldots, n+1, n)$ индуцирует чисто логтерминальное раздутие нашей особенности. Полученное многообразие лог-Фано $\left(E, \operatorname{Diff}{ }_{E}(0)\right)$ - это

$$
\left(x_{1}^{n}+\cdots+x_{n}^{n}+x_{n+1} \subset \mathbb{P}(1, \ldots, 1, n), \frac{n}{n+1}\left\{x_{n+1}=0\right\}\right)=\left(\mathbb{P}^{n-1}, \frac{n}{n+1} Q_{n}\right),
$$

где $Q_{n}$ - гладкая гиперповерхность в $\mathbb{P}^{n-1}$ степени $n$. По теореме 2.1 особенность слабо исключительна. Дивизор $\left\{x_{n+1}=0\right\}$ дает не чисто логтерминальное 1-дополнение. Поэтому особенность не исключительна. 


\section{СПИСОК ЦИТИРОВАННОЙ ЛИТЕРАТУРЫ}

[1] Prokhorov Yu.G. Blow-ups of canonical singularities // Algebra / ed. Yu. Bahturin. Proc. Internat. Conf. on the Occasion of the 90th Birthday of A. G. Kurosh (Moscow, Russia, May 25-30, 1998). Berlin: Walter de Gruyter, 2000. P. 301-317.

[2] Shokurov V. V. Complements on surfaces // E-print math.AG/9711024, 1997.

[3] Kollar J. et al. Flips and Abundance for Algebraic Threefolds. Astérisque. V. 211. Paris, 1992.

[4] Prokhorov Yu. G. Lectures on complements on log surfaces // E-print math.AG/9912111, 1999.

[5] Шокуров В. В. Трехмерные логперестройки // Изв. АН СССР. Сер. матем. 1992. Т. 56. № 1. C. $105-203$.

[6] Kawamata Y., Matsuda K., Matsuki K. Introduction to the minimal model program // Adv. Stud. in Pure Math. 1987. V. 10. P. 283-360.

[7] Ishii S. The quotient of log-canonical singularities by finite groups // Preprint, 1999.

[8] Fujino O. The indices of log canonical singularities // E-print math. AG/9909035, 1999.

[9] Prokhorov Yu. G., Ishii S. Hypersurface exceptional singularities // E-print math.AG/ 9910123, 1999.

Математический институт им. В. А. Стеклова

Поступило

E-mail : koudr@mi.ras.ru

10.10 .2000 